\title{
Skeletal muscle fibre type transformation following spinal cord injury
}

\author{
R Burnham ${ }^{1,2}$, T Martin ${ }^{3}$, R Stein ${ }^{4}$, G Bell ${ }^{3}$, I MacLean $^{3}$ and R Steadward ${ }^{2}$ \\ ${ }^{1}$ Glenrose Rehabilitation Hospital, Edmonton, Alberta; ${ }^{2}$ Rick Hansen Centre, University of Alberta; ${ }^{3}$ Faculty of \\ Physical Education and Recreation, University of Alberta and ${ }^{4}$ Division of Neurosciences, University of Alberta, \\ Canada
}

Following spinal cord injury (SCI), upper motor neuron paralysed muscles lose the normal type I (slow) and II (fast) fibre mosaic pattern and become predominantly composed of type II (fast glycolytic) fibres). The majority of the research demonstrating this fibre type shift was based on $\mathrm{pH}$ sensitive myofibrillar ATPase staining techniques on muscle from longstanding paraplegics and quadriplegics. The purpose of this study was to describe muscle fibre type changes over a wide time spectrum post SCI using immunofluorescent techniques which may be more sensitive to change. A total of 19 vastus lateralis muscle biopsy specimens were obtained from 12 SCI subjects representing time points of $0.5-219$ months post SCI. Fast and slow myosin heavy chain isoform distribution was determined on single muscle fibres for each of the biopsy specimens. Early post SCI $(<1$ month) myosin heavy chain $(\mathrm{MCH})$ isoform composition remained relatively stable. A transitional period was seen between 1 and 20 months post SCI wherein there was a progressive drop in the proportion of slow MHC isoform fibres and a rise in the proportion that co-expressed both the fast and slow MHC isoform. By approximately 70 months post SCI a new steady state had been reached characterized by almost exclusively fast MHC isoform expression. This research has demonstrated that post SCI muscle type II transformation occurs in stages and commences earlier than previously appreciated. Interventions aimed at preventing or minimizing the transformation would need to be instituted within weeks post SCI.

Keywords: spinal cord injury; skeletal muscle; histochemistry; myosin isoforms

\section{Introduction}

Following spinal cord injury (SCI) it is well documented in humans ${ }^{1-9}$ and animals ${ }^{10-12}$ that skeletal muscles below the level of an upper motor neuron lesion undergo marked changes in their morphological, metabolic, and contractile properties. Subsequent to SCI, paralyzed skeletal muscle generally becomes atrophic, possesses lower tension generating capacity and is less fatigue resistant. Muscle histochemical and metabolic profile shifts toward Type II (fast glycolytic) fibres have been well documented following SCI and may explain the problem of rapid muscle fatigability commonly encountered during rehabilitation efforts using functional electrical stimulation (FES). Knowing the time course of these adverse muscle changes is fundamental to timing interventions which may prevent or reverse the process. The remarkable plasticity of different properties of skeletal muscle makes the task of defining a temporal framework for the changes induced by SCI onerous. For example, it has been shown that some proteins (eg

Correspondence: Dr R Burnham, MD. FRCPC., c/o Glenrose Rehabilitation Hospital, 10230-111 Avenue, Edmonton, Alberta, Canada T5G 0B7 parvalbumin) ${ }^{13}$ protein systems (eg sarcoplasmic reticulum ATPase), ${ }^{14}$ or physiologic properties (eg twitch properties) $^{14}$ can be altered early in the face of perturbation to the muscle. Alternatively, other proteins (eg myosin), ${ }^{15,16}$ protein systems (myofibrillar ATPase),${ }^{16}$ or physiologic properties (eg fatigue resistance) ${ }^{17}$ are more stable. In this regard, the majority of the studies from SCI patients have relied on a relative comparison of the histochemically determined fibre types between normal and SCI muscle biopsies. ${ }^{1,2,6,7,9}$ The subjective nature of fibre typing based on saturated histochemical reaction products may be suited for muscle under steady state conditions, but is much less reliable under conditions of perturbation., ${ }^{5,18}$ Therefore, they may lack the sensitivity required to discern early changes in those properties determining fibre type. Further, most studies have used patients that have been SCI for a year or more. ${ }^{1,2,5,7-9}$ As such, they provide a valuable description of the long term consequences of SCI but provide little insight into the time course of those changes.

The present study reports on a logitudinal series of muscle biopsies from four SCI patients representing $0.5-3.5$ months post injury. In addition, data were 
obtained on eight single biopsies from patients at times ranging over $1-219$ months post SCI. Immunofluorescence techniques were used to determine the myosin heavy chain isoform composition of single muscle fibres. This muscle property was chosen because of its fundamental importance to muscle function and its well defined interrelationships with other muscle properties. $^{12,19}$ Further, the immunofluorescent techniques used provide a more sensitive index of change than was possible in previous studies on SCI muscle. Our findings suggest that fundamental muscle properties are altered much earlier than has been previously reported following SCI.

\section{Method}

Twelve subjects (eight males and four females) with a mean age of 22.4 years at the time of their biopsy signed informed consents to participate in this study. All had sustained traumatic spinal cord injury with complete upper motor neuron motor paralysis and sensory loss distal to the lesion level (C6-T8). Control data from 28 male and 18 female neurologically intact subjects of similar mean age from our laboratory are included for comparison.

Percutaneous vastus lateralis muscle biopsies were performed on each subject. Eight subjects had only 1 biopsy, whereas four subjects had serial biopsies. The serial biopsies were obtained over a time period of $0.5-3.5$ months post SCI. The subjects receiving only single biopsies represented a broader period of time post SCI (1-219 months). None of the legs which were biopsied were being trained with functional electrical stimulation, although the subjects from which serial biopsies were obtained were part of another study which involved FES and weight bearing of the contralateral limb. Previous reports of FES training applied unilaterally to a paralyzed limb demonstrated no crossover effect on the contralateral limb in SCI patients. ${ }^{20}$

Vastus lateralis muscle biopsies were performed under sterile technique without skin anesthetic using a $5 \mathrm{~mm}$ Bergstrom needle adapted for suction. ${ }^{21}$ The tissue was quickly mounted on cork with OCT embedding compound, rapidly frozen in isopentane cooled in liquid nitrogen, and stored at $-70^{\circ} \mathrm{C}$ for later analyses. Cryosections were cut at a thickness of $6 \mu \mathrm{m}$ at $-20^{\circ} \mathrm{C}$ using a Tissue-Tek Cryostat (Miles Laboratories), and mounted on chrome-gelatin $(0.1 \%$ gelatin, $0.01 \%$ chromium potassium sulfate) coated glass coverslips. The fast and slow myosin heavy chain isoform (MHC) distribution was determined in single muscle fibres using the immunofluorescence technique as described by Jiang et al. ${ }^{22}$ Briefly, the coverslips were incubated for $30 \mathrm{~min}$ in a blocking solution containing $2 \%$ bovine serum albumin and $2 \%$ rabbit serum in phosphate buffered saline (PBS), and then for $1 \mathrm{~h}$ with a mouse anti-chicken monoclonal antibody specific for slow MHC diluted 1: 10 in blocking solution (Stockdale, Stanford). This antibody is of the IgA subclass and, although it was raised to chick slow MHC, it cross reacts with human slow MHC. After thorough washing with PBS, primary antigen-antibody complexes were labelled by incubating for $1 \mathrm{~h}$ in rhodamine-conjugated goat anti-mouse immunoglobulin (goat anti-mouse IgA, TRITC labelled, Sera-Lab). The sections were then washed with PBS and incubated for $1 \mathrm{~h}$ with a moustanti rabbit monoclonal antibody specific for the fast MHC (MY-32, Sigma, St. Louis, MO). This antibody is of the IgG subclass and cross reacts with human fast MHC isoforms. The sections were washed again with PBS followed by a $1 \mathrm{~h}$ incubation with FITCconjugated goat anti-mouse IgG antibody (FITC-goat anti-mouse IgG, Zymed Laboratories, San Francisco, CA). The coverslips were finally mounted on slides using Krystalon (EM Diagnostic Systems Inc., Gibbstown, $\mathrm{NJ})$ mounting medium. Fluorescence was visualized on a Leitz Diplan microscope equipped with N2.1 and K2 filters. The N2.1 filter block incorporates an excitation band pass filter, allowing $515-560 \mathrm{~nm}$ wavelength light to pass and a suppression long-pass filter allowing wavelengths longer than $580 \mathrm{~nm}$ to pass in order to observe the TRITC label. The K2 filter block incorporates an excitation band pass filter, allowing $470-490 \mathrm{~nm}$ wavelength light to pass and a suppression long-pass filter allowing wavelengths of longer than $515 \mathrm{~nm}$ to observe the FITC label. The number of fibres expressing fast, slow or combined fast and slow MHC was determined from photographs in 50 to 80 fibres for each subject at each testing time (Figure 1).

\section{Results}

Table 1 contains the relative percentages of fibres in each SCI patient biopsy which expressed only the fast or slow MHC isoform, or expressed both isoforms simultaneously. In the control subjects' vastus lateralis, the vast majority $(98 \%)$ of fibres express only one MHC isoform with a slight predominance $(60 \%)$ of fibres having only the fast MHC. Only 2\% of the control muscle fibres demonstrated co-expression of both $\mathrm{MHC}$ isoforms. There were no gender differences. The significant trend toward the fast fibre type is clearly evident in the plot of Figure 2. In patients that had been SCI for over 70 months, the vast majority of fibres expressed only the fast $\mathrm{MHC}$ isoform. Thus, following long term SCI induced paralysis most fibres in the vastus lateralis take on a new steady state profile defined by a shift in the MHC expression to the fast isoform.

However, the shift to this new profile has interesting characteristics. In four of the six biopsies from patients that were obtained within one month of SCI there were no fibres identified that co-expressed the fast and slow MHC isoforms. In two other biopsies obtained at one month post SCI a minority of fibres (10 and 15\%) expressed both isoforms. Therefore, it appears that during the very early phase $(<4$ weeks) post SCI paralysis, the MHC isoform remains relatively stable. 


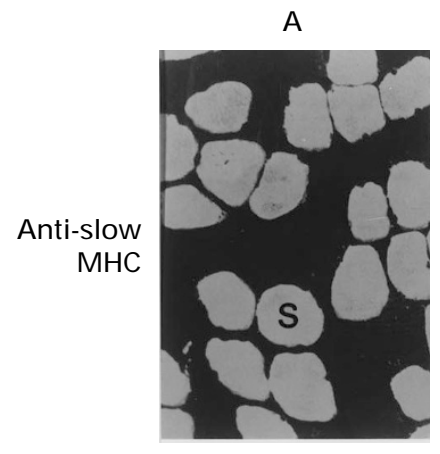

1 month post

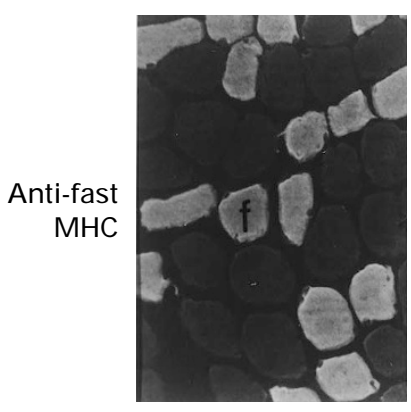

E
B

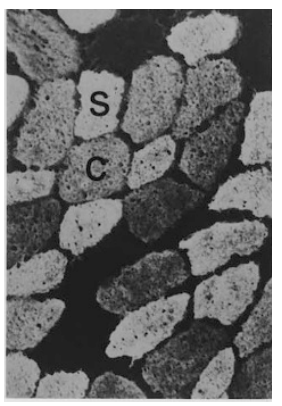

3 months post

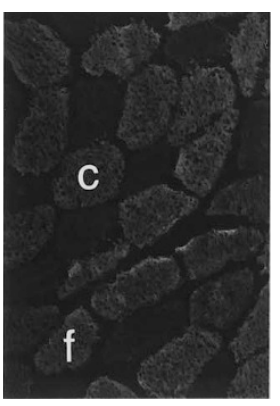

F

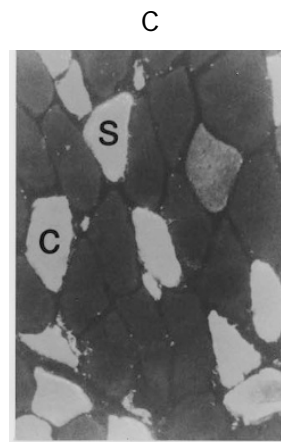

19 months post

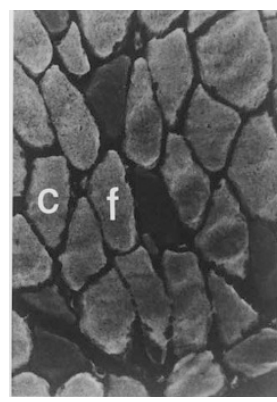

G

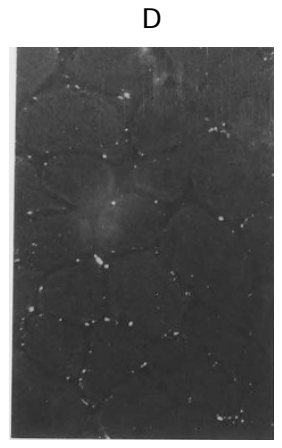

73 months post

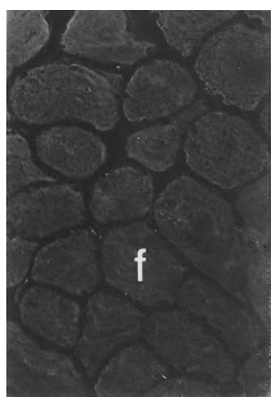

$\mathrm{H}$

Figure 1 Immunofluorescence labelling of paralysed vastus lateralis muscle 1, 3, 19 and 73 months post spinal cord injury demonstrating fibre proportion changes of slow (s), fast (f) and co-expressing (c) myosin heavy chain isoforms. A-D muscle labelled with anti-slow MHC (IgA) followed by rhodamine-conjugated anti-IgA. F-H identical sections showing anti-fast $\mathrm{MCH}(\mathrm{IgG})$ labelling visualized by fluorescein-conjugated anti-IgG

Table 1 Subject characteristics

\begin{tabular}{|c|c|c|c|c|c|c|c|}
\hline Patient & $\operatorname{Sex}$ & $\begin{array}{l}\text { Age at initial } \\
\text { biopsy (years) }\end{array}$ & Lesion & $\begin{array}{l}\text { Post injury } \\
\text { (months) }\end{array}$ & $\begin{array}{l}\text { Fast } M H C \\
(\%)\end{array}$ & $\begin{array}{c}\text { Slow } M H C \\
(\%)\end{array}$ & $\begin{array}{c}\text { Co-expressing } \\
(\%)\end{array}$ \\
\hline 1 & \multirow[t]{3}{*}{ M } & \multirow[t]{3}{*}{16} & \multirow[t]{3}{*}{$\mathrm{T} 5$} & 0.5 & 26 & 74 & 0 \\
\hline 1 & & & & 1.8 & 49 & 23 & 28 \\
\hline 1 & & & & 3 & 35 & 20 & 45 \\
\hline 2 & \multirow[t]{3}{*}{ M } & \multirow[t]{3}{*}{15} & \multirow[t]{3}{*}{$\mathrm{T} 8$} & 0.5 & 20 & 80 & 0 \\
\hline 2 & & & & 1.8 & 15 & 76 & 9 \\
\hline 2 & & & & 3.0 & 30 & 50 & 20 \\
\hline 3 & \multirow[t]{3}{*}{ M } & \multirow[t]{3}{*}{17} & \multirow[t]{3}{*}{ T6 } & 1 & 42 & 58 & 0 \\
\hline 3 & & & & 2.5 & 37 & 23 & 40 \\
\hline 3 & & & & 3.5 & 38 & 24 & 38 \\
\hline 4 & \multirow[t]{2}{*}{ M } & \multirow[t]{2}{*}{29} & \multirow[t]{2}{*}{$\mathrm{T} 8$} & 1 & 48 & 52 & 0 \\
\hline 4 & & & & 2 & 34 & 62 & 4 \\
\hline 5 & $\mathrm{M}$ & 29 & $\mathrm{~T} 4$ & 1 & 31 & 59 & 10 \\
\hline 6 & $\mathrm{~F}$ & 17 & $\mathrm{C} 8$ & 1 & 40 & 45 & 15 \\
\hline 7 & $\mathrm{M}$ & 28 & $\mathrm{C} 8$ & 19 & 52 & 2 & 46 \\
\hline 8 & $\mathrm{M}$ & 23 & C7 & 20 & 66 & $2 \overline{6}$ & 8 \\
\hline 9 & $\mathrm{M}$ & 24 & $\mathrm{~T} 4$ & 73 & 100 & 0 & 0 \\
\hline 10 & $\mathrm{~F}$ & 23 & T5 & 78 & 97 & 3 & 0 \\
\hline 11 & $\mathrm{~F}$ & 27 & C6 & 86 & 100 & 0 & 0 \\
\hline 12 & $\mathrm{~F}$ & 35 & $\mathrm{C} 7$ & 219 & 85 & 14 & 1 \\
\hline \multirow{2}{*}{\multicolumn{5}{|c|}{$\begin{array}{l}\text { non SCI control male (mean } \pm \text { s.d.) } \\
\text { non SCI control female (mean } \pm \text { s.d.) }\end{array}$}} & $60 \pm 5$ & $38 \pm 5$ & $2 \pm 1$ \\
\hline & & & & & $57 \pm 3$ & $41 \pm 4$ & $2 \pm 1$ \\
\hline
\end{tabular}




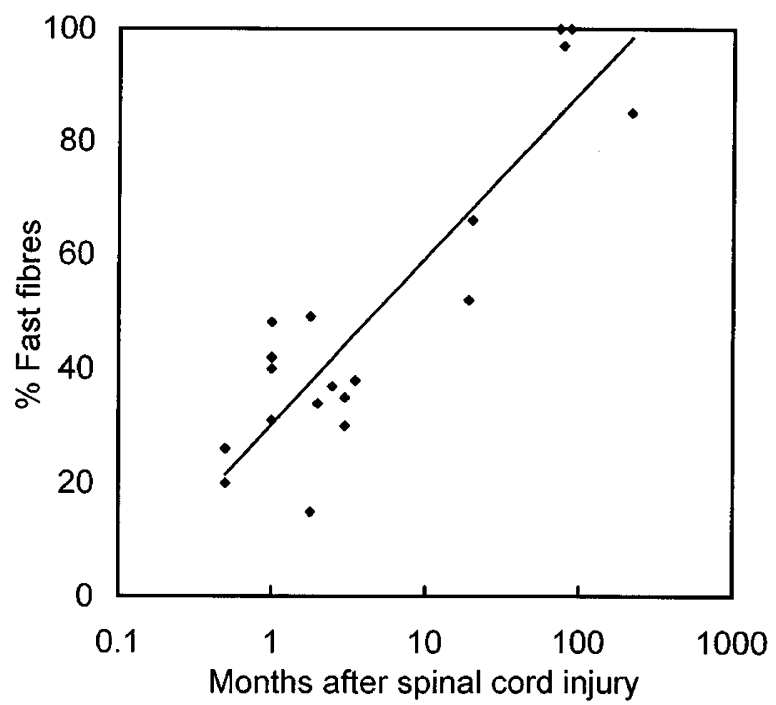

Figure 2 The percentage of fast fibres increases with time after spinal cord injury. The straight line was fitted by the least mean squares method on this semilogarithmic plot

In the data obtained from patients between 1-20 months post SCI, there is an increase in the relative proportion of fibres that co-expressed the fast and slow MHC isoform commencing between the first and second months post SCI. Thus, fibres undergo a transitional period during which they alter the expression of their MHC isoform presumably by down-regulation of the slow MHC and up-regulation of the fast MHC isoform. As such, there is a period of time where a single fibre may express both MHC isoforms simultaneously, prior to achieving the new steady state characterized by a predominance of fibres expressing only the fast MHC isoform. Although we have no information about the state of the muscle between 20 and 73 months post injury, it is apparent that a new steady state is reached by the 73 month post injury point. The kinetics of this process can be modelled, as described in the Appendix and shown in Figure 3. Essentially, there are three states in which fibres express only fast, only slow or both MHC isoforms. The best fitting curves in Figure 3 assume that initially $66 \%$ of the fibres in the muscle are slow and that there is a transition with a time constant of 4.7 months to the co-expressing state, and a further time constant of 17 months to the fast state. This model accounts for nearly $80 \%$ of the variance in the data, leaving only $20 \%$ due to variations between individuals and measurement or sampling errors. Figure 1 displays representative biopsy specimens over four time periods post SCI.

\section{Discussion}

The present study was undertaken in order to describe changes in the MHC isoform content of single muscle
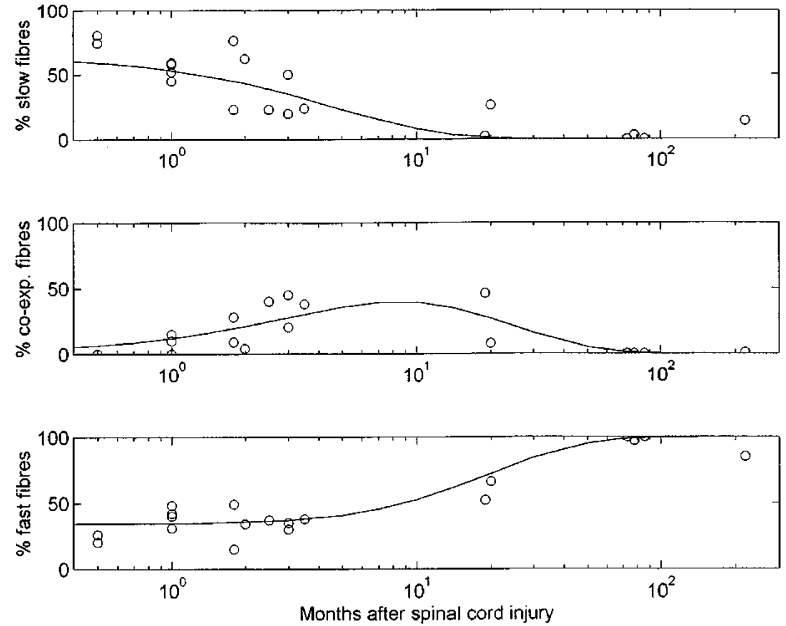

Figure 3 The percentage of slow fibres (top), fast fibres (bottom) and fibres co-expressing both slow and fast MHC can be well fitted by a simple model that is derived in the Appendix. The fitted curves account for nearly $80 \%$ of the variance in the data

fibres following SCI induced upper motor neuron paralysis. We obtained data over a wide range of post SCI time periods $(0.5-219$ months) to discern how rapidly a fundamental muscle property (MHC isoform) was altered by SCI. The MHC isoform of a fibre was chosen as a property index because of the following reasons: (1) Nearly all adult normal muscle fibres express only one MHC isoform; ${ }^{23}$ (2) The isoform composition of adult normal muscle appears to be stable, ${ }^{23}$ (3) In normal adult muscle the MHC isoform is related to the standard histochemical based fibre typing classification criteria predicated on the $\mathrm{pH}$ sensitivity of myofibrillar ATPase and histochemically determined Type I fibres have the slow MHC isoform, Type II fibres and fast MHC isoform, and Type C fibres co-express both MHC isoforms: ${ }^{24}$ (4) The MHC isoform has been shown to have predicable interrelationships with other muscle properties under control conditions and in various models of muscle perturbation, ${ }^{12,19}$ and, (5) Immunofluorescence techniques are available for the determination of the $\mathrm{MHC}$ isoform composition of single fibres. ${ }^{22}$ This provides a sensitive method by which to study changes in this muscle property after SCI. Human muscle has at least three different $\mathrm{MHC}$ isoforms. ${ }^{18}$ In this study we made no attempt to differentiate the subpopulations of the fast MHC isoforms.

Several previous reports are available on the effects of SCI induced paralysis on human muscle. In each case they have used standard qualitative histochemical methods to fibre type muscle biopsies at various times post SCI. In the majority of cases these studies have used patients that have been SCI for nearly one or more years. Grimby et $a l^{1}$ reported that in SCI patients 10 months -10 years post injury the vastus 
lateralis, gastrocnemius, and soleus muscles were predominately composed of Type II fibres. Stilwill and Sahgal $^{2}$ reported that in the quadriceps (specific muscle not identified) of three patients at least 6 months post injury the fibre type composition remained unchanged in two patients while in the third there was a predominance of Type II fibres. In the tibialis anterior of patients $2-11$ years post SCI, Martin et $a l^{5}$ reported a marked increase in the relative proportion of Type II fibres compared to control. Patients studied 15-24 months post SCI by Greve et $a l^{6}$ showed that in the vastus lateralis of three of four patients, Type II fibres were the most prevalent while in one patient the majority were Type I fibres. Round et $a l^{7}$ determined that biopsies from the vastus lateralis of patients 11 months -9 years post SCI were composed primarily of Type II fibres. In a recent report by Rochester et $a l^{8,9}$ patients were studied $1-14$ years post SCI. In six of seven patients there was a predominance of Type II fibres found in biopsies from the tibialis anterior. In one patient the relative proportion of Type I and II fibres was unchanged from control. Taken in total, these previous studies illustrate that longstanding paralyzed muscle appears to undergo a shift in its histochemical fibre type profile toward that of a predominance of Type II fibres. In fact, this transformation in some patients appears to be complete with no Type I fibres evident in the biopsy. These data are essentially corroborated by our findings that the patients with SCI greater than 70 months had fibres which primarily expressed only the fast MHC isoform. Therefore, it appears that following prolonged upper motor neuron paralysis secondary to SCI, muscle fibres that previously had Type I or slow twitch properties alter their phenotypic expression to become Type II or fast twitch.

Two studies from Scelsi and collaborators ${ }^{3,4}$ are of particular relevance to the present study because they also contain data from SCI patients over significantly earlier time periods post injury than the aforementioned studies. Scelsi et $a l^{3}$ reported that biopsies from the rectus femoris of SCI patients $1-17$ months post injury showed what they described as progressive stages of change. The tissue was analyzed using standard qualitative histochemical techniques. Biopsies obtained 1-4 months post SCI showed no change in the relative percentage of Type I and II fibres; however, atrophy was seen particularly in the Type II fibres. Biopsies obtained 4-9 months post SCI showed atrophy in both fibre types with a reduction in the relative percentage of Type I fibres. In the biopsies studied 10-17 months post SCI the Type I fibres continued to show atrophy and there was a marked increase in the relative percentage of Type II fibres and concomitant decrease in the percentage of Type I fibres. A later study ${ }^{4}$ looked at the changes in the medial gastrocnemius and soleus muscles in patients 1-10 months post SCI. The MHC content of the whole biopsy (in contrast to single fibre analysis used in the present study) was estimated by electrophoretic separation techniques along with the muscle fibre typing. The latter study concluded that in the initial $1-6$ months of paralysis the primary change was atrophy primarily of the Type II fibres with no change in the relative percentage of fibre types or MHC content. However, after 8 months there was atrophy in both fibre types with an increase in the relative percentage of Type II fibres and fast MHC content. In summary, these two studies suggest that the earliest changes post SCI are to the morphological muscle properties and that only after approximately 7-8 months is the shift of the Type I to Type II fibres manifested.

Our findings support the concept of progressive stages of change in SCI paralyzed muscle introduced by Scelsi et al. ${ }^{3,4}$ However, using the sensitive technique of single fibre MHC isoform determination, the onset of change appears to be earlier than previously described. Our data suggest that changes in the fundamental properties of muscle probably occur as early as $4-6$ weeks post injury. The time constant of 4.7 months indicates that $62 \%$ of the fibres will have undergone a transformation over this period of time. Further, it appears that as the Type I fibres change to Type II they undergo a transitional phase where they co-express slow and fast MHC isoforms. This most likely signifies the downregulation of the slow $\mathrm{MHC}$ isoform and the upregulation of the fast $\mathrm{MHC}$ isoform in those fibres. The mechanism may be similar to that reported to occur following chronic low frequency electrical stimulation of muscle where Type II fibres are transformed toward Type I. A significant increase in Type C fibres which coexpress slow and fast $\mathrm{MHC}$ isoforms was seen in this FES model in rabbits. ${ }^{24}$

Currently the mechanism(s) responsible for the alterations in muscle that occur following SCI are unknown. However, it does appear that not only do the fibres atrophy rapidly post $\mathrm{SCI}^{3,4}$ but also they begin to change their fundamental properties early post injury. From a rehabilitation perspective it would be prudent to initiate therapeutic interventions aimed at attenuating the adverse muscle impact of SCI as early as safely possible. In this regard, research is currently underway to evaluate the impact of weightbearing combined with functional electrical muscle stimulation early post SCI on muscle and bone.

\section{Acknowledgements}

The authors thank Dr. Frank Stockdale of Stanford University for generously providing the mouse antichicken monoclonal antibody S58 and the Glenrose Rehabilitation Hospital Research Fund for providing funding for the project.

\section{References}

1 Grimby G, Broberg C, Krotkiewska I, Krotkiewski M. Muscle fibre composition in patients with traumatic spinal cord lesion. Scand J Rehab Med 1976; 8: 37-42. 
2 Stilwill EW, Sahgal V. Histochemical and morphologic changes in skeletal muscle following cervical cord injury: a study of upper and lower motor neuron lesions. Arch Phys Med Rehabil 1977; 58: $201-206$.

3 Scelsi R et al. Muscle fibre type morphology and distribution in paraplegic patients with traumatic cord lesions. Acta Neuropathol 1992; 57: $243-248$

4 Lotta S et al. Morphometric and neurophysiological analysis of skeletal muscle in paraplegic patients with traumatic cord lesion. Paraplegia 1991; 29: $247-252$

5 Martin TP, Stein RB, Hoeppner PH, Reid DC. Influence of electrical stimulation on the morphologic and metabolic properties of paralyzed muscle. J Appl Physiol 1992; 72: 1401 - 1406.

6 Greve JMD et al. Functional electrical stimulation (FES): muscle histochemical analysis. Paraplegia 1993; 31: $764-770$.

7 Round JM, Barr FMD, Moffat B, Jones DA. Fibre areas and histochemical fibre types in the quadriceps muscles of paraplegic subjects. J Neuro Sci 1993; 116: 207-211.

8 Rochester L et al. Influence of electrical stimulation of the tibialis anterior muscle in paraplegic subjects. I. Contractile properties. Paraplegia 1995; 33: 437 - 449 .

9 Rochester L et al. Influence of electrical stimulation of the tibialis anterior muscle in paraplegic subjects 2. Morphological and histochemical properties. Paraplegia 1995; 33: 514-522.

10 Leiber RL et al. Long-term effects of spinal cord transection on fast and slow skeletal muscle I: contractile properties. Exp Neurol 1986; 91: $423-434$.

11 Leiber RL et al. Long-term effects of spinal cord transection on fast and slow rat skeletal muscle II: morphometric properties. Exp Neurol 1986; 91: 435-448.

12 Roy RR, Baldwin KM, Edgerton VR. The Plasticity of Skeleta Muscle: Effects on Neuromuscular Activity. In Exercise and Sport Reviews. (Ed.) J Holloszy. Baltimore: Williams and Wilkins, 1991 Vol 19: pp 269-312.

\section{Appendix}

Figure 1 in the Results shows the trend to the MHC characteristic of fast fibres clearly, but does not describe the kinetics of the process. Here we assume a simple model with three states: fast (f), slow (s) and co-expressing (c). There will be two time constants, one (a) as the fast MHC is upregulated, and a second (b) as the slow MHC is downregulated. The model assumes that eventually all fibres will show only the fast $\mathrm{MHC}$; i.e. there are no back rate constants. This model can be expressed as:

$$
\begin{gathered}
a \quad b \\
s \rightarrow c \rightarrow f
\end{gathered}
$$

Solving the kinetic equations by standard methods gives:

$$
\mathrm{s}=\mathrm{s}_{\mathrm{o}} \exp (-\mathrm{at})
$$

13 Klug G, Reichmann H, Pette D. Rapid reduction in parvalbumin concentration during chronic stimulation of rabbit fast twitch muscle. FEBS Lett 1983; 152: 180-182.

14 Heilmann C, Pette D. Molecular transformations in sarcoplasmic reticulum of fast twitch muscle by electrostimulation. Eur $J$ Biochem 1979; 93: 437 - 446.

15 Brown WE, Salmons S, Whalen RG. The sequential replacement of myosin subunit isoforms during muscle type transformation induced by long term electrical stimulation. J Biol Chem 1983; 258: $14,686-14,692$.

16 Salmons S, Sreter FA. Significance of impulse activity in the transformation of skeletal muscle type. Nature 1976; 263: 30 - 34

17 Martin TP, Bodine-Fowler SC, Edgerton VR. Coordination of electromechanical and metabolic properties of cat soleus motor units. Am J PHysiol 1988; 255: C684-C693.

18 Talmadge RJ, Roy RR, Edgerton VR. Muscle fibre types and function. Curr Opin Rheumatol 1993; 5: 695-705.

19 Roy RR et al. Interrelationships of contraction time, Vmax, and myosin ATPase after spinal transection. J Appl Physiol 1984; 56: $1594-1601$

20 Stein RB et al. Optimal stimulation of paralyzed muscle after human spinal cord injury. J Appl Physiol 1992; 72: 1393-1400.

21 Bergstrom J. Percutaneous needle biopsy of skeletal muscle in physiological and clinical research. Scand J Clin Lab Invest 1975; 35: $609-616$

22 Jiang B, Roy RR, Edgerton VR. Expression of fast fibre enzyme profile in the cat soleus after spinalization. Muscle Nerve 1990; 13: $1037-1049$.

23 Biral D, Betto R, Danielli-Betto D, Salviati G. Myosin heavy chain composition of single fibres from normal human muscle. Biochem J 1988; 250: 307 -308.

24 Staron RS, Pette D. Nonuniform myosin expression along single fibres of chronically stimulated and contralateral rabbit tibialis anterior muscle. Pflugers Arch 1987; 409: 67-73.

$$
\begin{gathered}
c=s_{o} a[\exp (-a t)-\exp (b t)] /(b-a) \\
f=1-s-c
\end{gathered}
$$

where $s_{0}$ is the percentage of fibres that initially express the slow MHC.

To optimize the fit a gradient search algorithm was used. Initial values of $\mathrm{a}, \mathrm{b}$ and $\mathrm{s}_{0}$ were selected and each parameter was varied by a fixed percentage $(50 \%)$. The change that reduced the error between the data points and the fitted values was determined and the process repeated until no change of this magnitude would reduce the error. Then the percentage was halved and the process repeated until the percentage was reduced to less than $1 \%$. Figure 2 shows the data for all SCI patients and the fitted curve for the best fitting model. 